\title{
LES of the Flow in a Rotating Rib-Roughened Duct
}

\author{
D.Borello, A. Salvagni, F. Rispoli, and K. Hanjalic
}

\section{Introduction}

In gas turbine cooling internal blade channels are often lined with rib turbulators. These are turbulence promoters that enhance the heat transfer between the blade (hot) surface and the cooling air flowing inside the channel. The rib orientation w.r.t the mainstream flow causes very complex phenomena including unsteadiness, boundary layer separation and reattachment, recirculation, streamlines curvature. To improve the current internal cooling designs, detailed flow field (and heat transfer) predictions are needed to detect the presence of critical region were hot spots can develop leading to a material failure. The flow features inside a stationary ribbed channels was largely analized. However, to analyze the typical features of the flows in turbine rotors, influence of rotation must be considered. Rotation introduces a background vorticity leading to a flow stabilisation on the trailing side and a flow destabilisation on the leading side. Rotation number (Ro) is introduced, $\mathrm{Ro}=\Omega \mathrm{D} / \mathrm{U}$, where $\mathrm{U}$ is the bulk velocity, $\mathrm{D}$ the hydraulic diameter of the duct and $\Omega$ is the angular velocity. Recently, Coletti and co-workers carried out extensive experimental PIV analysis on a rib-roughened channel in rotating conditions [1]. They investigated the influence of rotation in a rib-roughened channel containing 10 ribs equally spaced. The rotational axis is orthogonal to the rib-roughened surface. The test section and instrumentation were mounted on a disk rotating in a direction that is perpendicular to the main flow. Direct flow measurement were carried out when the disk was rotating. The wallnormal pressure gradient is modified to balance the Coriolis forces. This has

D.Borello · A. Salvagni · F. Rispoli

Dipartimento di Ingegneria Meccanica e Aerospaziale, 'Sapienza' Universit di Roma, Italy, e-mail: domenico.borello - alessandro.salvagni - franco.rispoli@uniroma1.it

K.Hanjalic

Novosibirsk State University, Russia, e-mail: khanjalic@gmail.com 
a strong effect on the boundary layer that is stabilizing in case of clockwise rotation and a de-stabilizing in case of a counter-clockwise rotation. Here we reproduced the same conditions of Coletti et al. [1] for the anti-clockwise rotation, by performing a well-resolved Dynamic Smagorinsky LES.

\section{Flow specification and computational details}

The channel has an aspect ratio equal to 0.9 and the ribs generate a blockage factor of $0.1, \mathrm{~h}$ is the rib height. The Navier-Stokes equations system was solved in dimensionless variables. As reference vaules we used duct hydraulic diameter, bulk flow velocity and air properties at $20 \mathrm{C}$. The ensuing Reynolds number is equal to 15,000 . As stated by Coletti et al. [1], after the 6 th rib the flow can be considered periodic. Then only one section between two successive ribs subjected to anti-clockwise rotation with $\mathrm{Ro}=0.3$ is considered here. Non-rotating solution was computed for comparisons. The rotation axis (and origin of axis) was placed in the corner region behind the rib. The well-validated unstructured Finite Volume code T-FlowS is used for the present case. The code was previously successfully used by some of the authors for LES computations [2]. The computational domain was meshed using a block-structured hexaedral ortogonal grid with about $6.5 \mathrm{M}$ cells. All the wall neighbouring cells have a $\mathrm{y}+$ value less than 0.5 . A coarser grid with $3 \mathrm{M}$ cells was considered for comparisons.

A Crank-Nicolson/CDS scheme was adopted to obtain a second-order accurate solution. Fully coupled solution of the Navier-Stokes system was obtained using the SIMPLE scheme, while the Preconditioned BiCG solver is adopted for the solution of the algebraic linearized equation system. Periodic boundary conditions were imposed at the inlet and outlet surface in streamwise conditions. On the solid walls no-slip conditions were set. The non dimensional time step was selected equal to $5 \mathrm{e}-4$. The maximum CFL number is smaller than 0.3. The computations were performed on the CRESCO SP5 cluster at Casaccia (ENEA) using 32 processors. On such a computer 25 seconds are required for performing 1 time step for both computations on the fine grid. Computations were performed for 2 flow through times (FTT) before starting to collect statistics. In Table 1 the time averaging periods are summarized.

Table 1 Time averaging period

\begin{tabular}{lll}
\hline Case & Coarse Grid & Refined Grid \\
\hline No Rotation & 11 FTT & 13 FTT \\
Anti-clockwise rotation & 22 FTT & 15 FTT \\
\hline
\end{tabular}




\section{Results}

To analyze the influence of Coriolis forces on the mean flow, streamlines are plotted for the rotating and the non-rotating simulations in Figure 1.
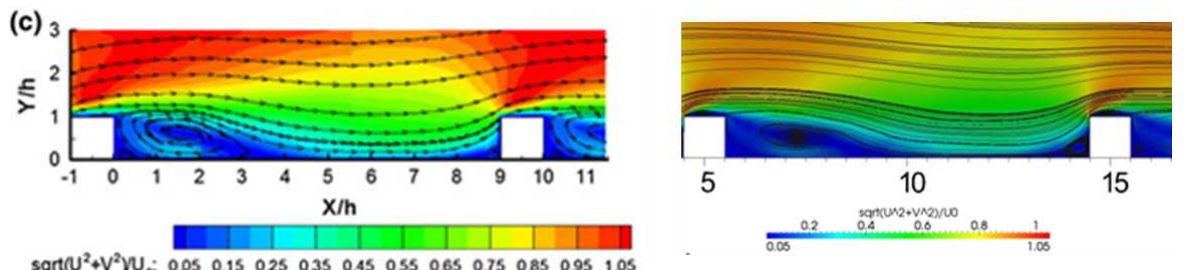

Fig. 1 Streamlines for the rotating case - left: exp; right: LES

In both cases, the presence of the rib generates a large recirculation bubble. The anti-clockwise rotation enhance the entrainment of the separated region, leading to a pressure reduction in the separated region. This stronger pressure gradient induces a stronger streamlines deviation and to a reduction in the lenght of the separation bubble. This is in agreement with the results of Coletti et al. [1]. The analysis of the pressure gradient plots (not shown here) confirms these findings. It is important to note that, when using the coarse grid the quality of the predictions deteriorated (Table 2).

Table 2 Lengh of recirculation bubble

\begin{tabular}{llll}
\hline Case & Coarse Grid & Refined Grid & Exp \\
\hline No Rotation & $3.90 \mathrm{~h}$ & $3.90 \mathrm{~h}$ & $3.85 \mathrm{~h}$ \\
Anti-clockwise rotation & $3.85 \mathrm{~h}$ & $3.50 \mathrm{~h}$ & $3.45 \mathrm{~h}$ \\
\hline
\end{tabular}

Coriolis force also affects the other vortical structures that are present close to the rib. As a matter of fact the downstream corner vortex generated by the large recirculation bubble is shrinked and the upstream corner vortex is enlarged. Furthermore, the recirculation bubble generated on the top of the rib is affected by the curvature of the streamlines passing above the rib. Due to the Coriolis effects the recirculation bubble is thicker and then the streamlines has a lower curvature when passing above the rib. Then, the rib recirculation bubble become thicker than in the non-rotating case and extends for all the rib (Figure 2).

The plots of axial velocity downstream from the rib demonstrates that a fair agreement is obtained between the experiments and LES results, especially when considering thickness of recirculation bubble (see Figure 3). 

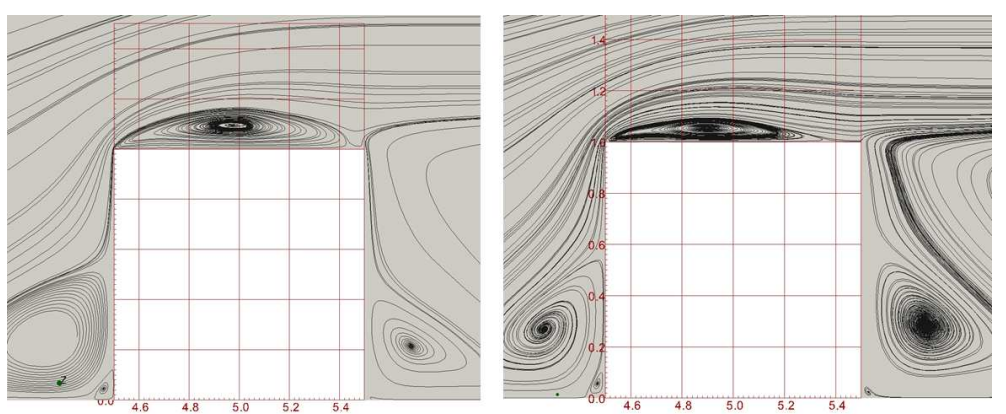

Fig. 2 Vortical structures around the rib - left: anti-clockwise rotation; right: non-rotating
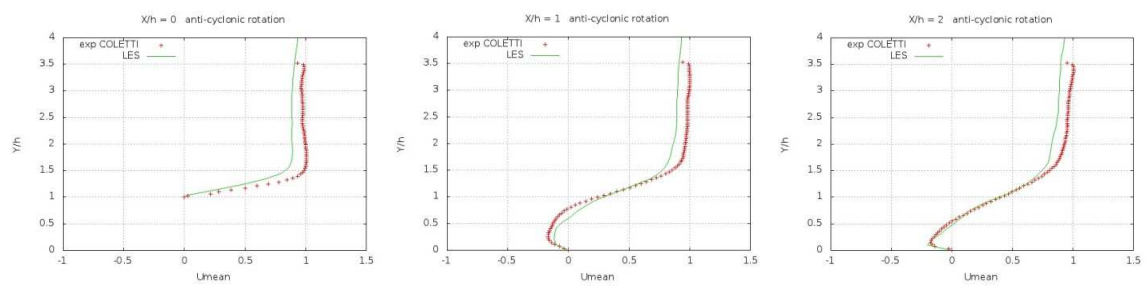

Fig. 3 Streamwise velocity downstream from the rib - left: $\mathrm{x} / \mathrm{h}=0$; center: $\mathrm{x} / \mathrm{h}=1$; right: $\mathrm{x} / \mathrm{h}=2$ (anti-clockwise rotation case)

Figure 4 shows that the rib is the main source of shear stress and the presence of rotation enhance the production of uv. Over the rib the shear stress is suppressed due to the low turbulence level in the slow recirculation bubble.

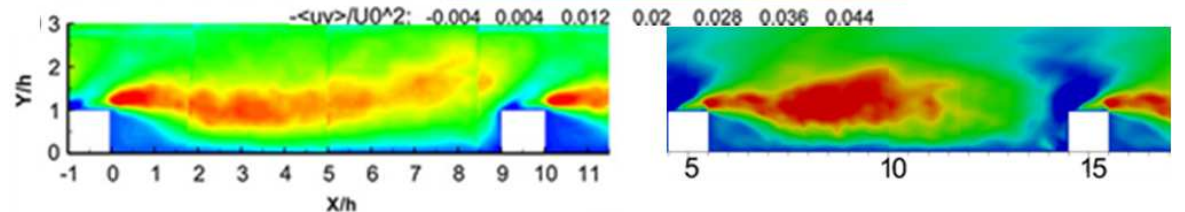

Fig. 4 Shear stress -uv (anti-clockwise rotation case): left - exp, right - LES

The influence of the lateral walls on the flow field were out of the scope of the PIV (referring only to the mid-plane). It is then interesting to investigate the influence of rotation in planes that are parallel to the rib-roughened wall. In particular, following the analysis of Casarsa et Arts [3] carried out on a nonrotating rib-roughened channel with high blockage ratio (not shown here), we analyze the streamlines plot in two planes: at $0.05 \mathrm{~h}$ and $0.5 \mathrm{~h}$ far from the 
wall (Figure 5$)$. On the first plane $(0.05 \mathrm{~h})$ it is possible to see the presence of the vortical structures discussed before: upstream corner vortex (clockwise rotation), downstream corner vortex (anti-clockwise) and the recirculation bubble extending for about 0.3 of the space between two consecutive ribs. In the non rotating case, when moving towards the lateral walls, the recirculation bubble lenght is strongly reduced and then its footprint of this plane has a bow shape. Notably, this effect is less evident in the rotating case due to the cited shrinking of the recirculation bubble that is particularly relevant in the mid-plane. The section at $\mathrm{y} / \mathrm{h}=0.5$ shows some differences: first of all the two small vortices close to the rib are not existing at this distance from the wall (see also Figure 2). Furthermore, two pitchwise vortical structures are present close to the lateral walls immediately downstream from the rib. Again, the bow shape of the recirculation bubble is somewhat suppressed in the rotating case.
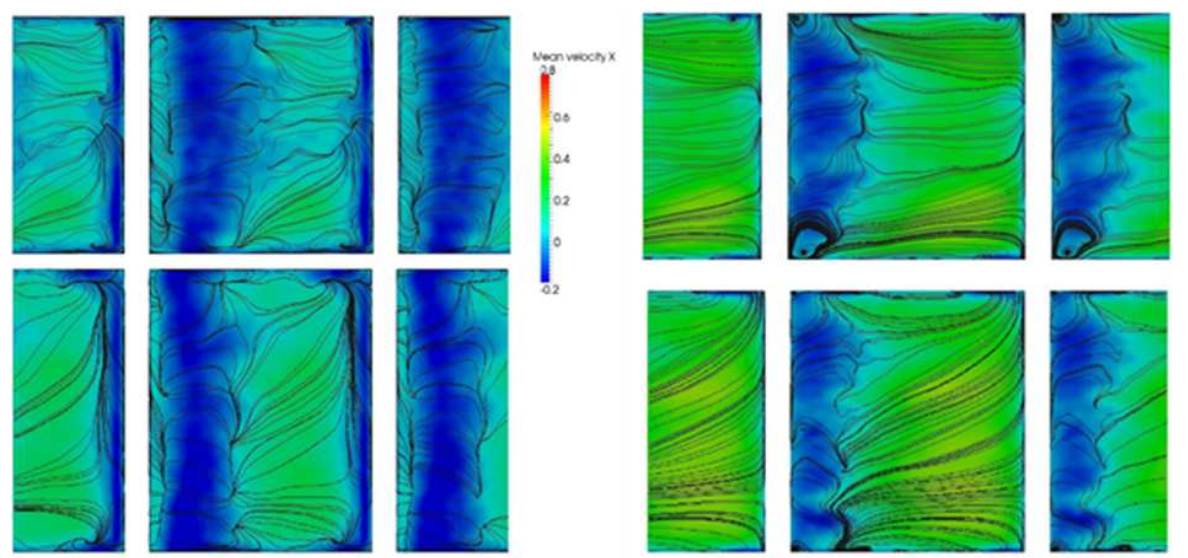

Fig. 5 Streamlines on the rib-roughened wall parallel plane; left: $0.05 \mathrm{~h}$; right: $0.5 \mathrm{~h}$; up: non rotating; down: anti-clockwise roating

Two Coriolis induced secondary flows extending for all the section are present in the rotating case. Such structures push the fluid from the central part of the duct towards the lateral walls. Therefore the streamlines are swept towards the rib-roughened wall in the mid-plane and in opposite direction when moving close to the lateral walls (see Figure 6)

Finally, in Figure 7 the instantaneous velocity plots are presented. In the non-rotating case the velocity is not perturbed by the rib presence for $y$ greater than 2.5. On the opposite, in the rotating case, the vortical structures extend for about half of the channel height, suggesting that a more vigorous heat removal is expected in the rotating case. 

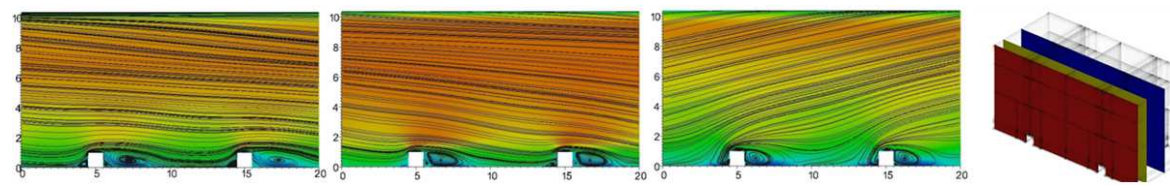

Fig. 6 Streamlines along pitchwise planes ; left: mid-pitch; center: 0.15 pitch; right: 0.05 pitch
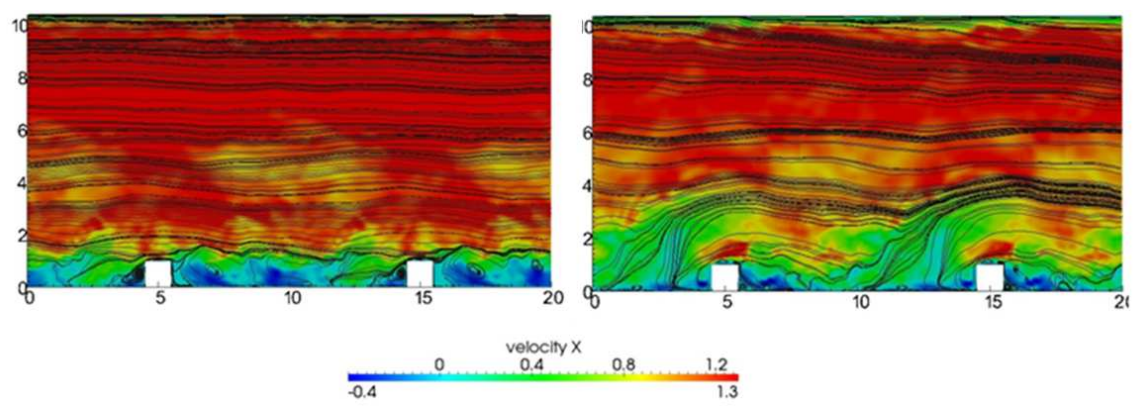

Fig. 7 Unsteady streamlines along the mid-pitch plane; left: non-rotating; right: anticlockwise rotation

\section{Conclusions}

LES of the flow in a rib-rougheded channel subjected to anti-clockwise rotation was carried out. The turbulent flow structures induced by the geometry and the rotation were identified and discussed. In particular, we noticed the presence of large Coriolis-induced secondary flows and the change in the bow shape of the recirculation bubble when considering an anti-clockwise rotation. The presence of rotation induced a destabilizing effect increasing the turbulence and flow unsteadiness.

\section{References}

1. Coletti, F., Maurer, T., Arts, T., Di Sante, A.: Flow Field Investigation in a Rotating Rib-Roughened Channel by means of Particle Image Velocimetry. Exp Fluids 52, 1043-1061 (2012)

2. Delibra, G., Borello, D., Hanjalic, K., Rispoli, F.: LES of flow and heat transfer in a channel with a staggered cylindrical pin matrix. In: V. Armenio, B. Geurts and J. Froelich (Eds), Direct and Large-eddy Simulation VII, (Proc. 7th Int. ERCOFTAC Workshop, Trieste, Sept 9-10, 2008), Springer Science and Media B.V.

3. Casarsa, L., Arts, T.: Experimental Investigation of the Aerothermal Performance of a High Blockage Rib-Roughened Cooling Channel. J. Turbomach. 127, 580-588 (2005) 